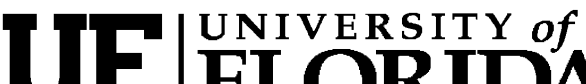 FLORIDA \\ IFAS Extension
}

CIR 1436

\section{Enhancing Natural Resource Programs: Designing Effective Brochures ${ }^{1}$}

\section{Martha C. Monroe and Ludovica Weaver ${ }^{2}$}

Natural resource programs share information, educate citizens, and inspire action. Across Florida, a variety of programs build public support for protecting groundwater, reducing waste, conserving energy, removing invasive exotics, stewarding resources, and restoring wildlife habitat. These programs use a variety of education and communication strategies to provide the audience with appropriate messages, learning opportunities, and skills. Sometimes this information is best shared through a brochure.

Brochures are an established format for sharing important written information with the public. They are small enough to take home and tape to the refrigerator. They can be reproduced in large quantities and mailed to a target audience. They can be designed with graphics, lines, and text to make a significant amount of information easy to interpret. Brochures are a versatile communication tool.

Brochures can provide background information about a topic, such as the coral reefs of Florida, but are more often used to spell out action steps that people should take. Since they can be picked up by an audience and studied at a later time, brochures can provide detailed procedural steps, such as how to prune landscape plants in fire-prone ecosystems, how to remove Brazilian pepper, or how to test well water. They can also advertise an upcoming program; some brochures may include a tear-off registration or reply form.

The brochures depicted in Figure 1 support a festival, an institute, and landscaping tips.

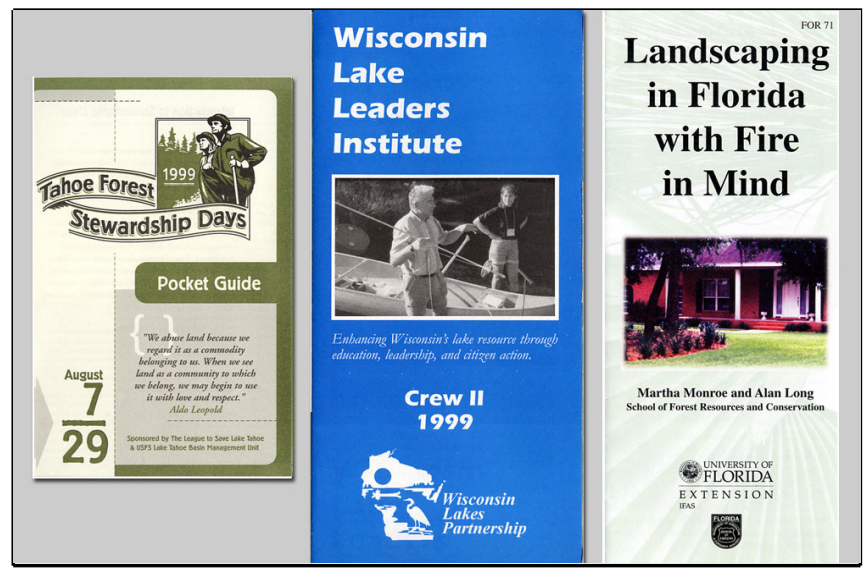

Figure 1. The brochures depicted in Figure 1 support a festival, an institute, and landscaping tips.

1. This document is CIR 1486, one of a series of the School of Forest Resources and Conservation, Florida Cooperative Extension Service, Institute of Food and Agricultural Sciences, University of Florida. First published: April 2007. Please visit the EDIS Web site at http://edis.ifas.ufl.edu.

2. Martha C. Monroe, Associate Professor, and Extension Specialist, School of Forest Resources and Conservation, University of Florida, Gainesville, FL 32611. Ludovica Weaver, Graphic Designer, University of Florida, Gainesville, FL 32611.

The use of trade names in this publication is solely for the purpose of providing specific information. UF/IFAS does not guarantee or warranty the products named, and references to them in this publication does not signify our approval to the exclusion of other products of suitable composition. 
This document provides a framework for the design of a brochure. It lists a variety of questions that should help guide the development of your brochure. It also spells out the procedures in Microsoft Word $2000^{\circledR}$ to accomplish the formatting suggestions. Since there are many effective brochure designs, and no single best way to produce one, this document has suggestions and tools to enable you to create a unique masterpiece.

\section{Brochure message}

An effective brochure has all the elements on the page working together; the paper size, color, orientation, pictures, design, and text all communicate the same message. By starting with a clear intent of what will be communicated in the brochure, you can more easily make design decisions. When all the elements reinforce each other, the brochure has a clear message.

This also makes it challenging to design a brochure, because each decision impacts other ones. Brochures with two ink colors can handle designs different from those with one color. Paper size affects the number of panels. Purpose affects orientation. Although this guide lists these decisions one after the other, experienced brochure designers usually consider them concurrently. As you work through this guide, you may want to modify earlier decisions and change the overall design of your brochure.

\section{Identify the purpose}

What are you communicating? What is the main goal of the brochure, and what should readers know and be able to do? If the intent is to inform, consider wide columns with a lot of text and pictures. If you are conveying a procedure, the general layout may flow from step to step with narrow columns, which create more flexibility for the text.

Will the brochure have a response card for a mailer? Usually this item is placed on the far right panel. Consider how this fits the overall design of the brochure. Make sure that essential information about the event is not on the reverse side of the response panel. Are there any design elements (e.g., lines, colors, images) that could be carried through the brochure to unify the whole piece? Symbols or lines may help.

Once you have answered these questions, envision how this information might take form by making little sketches of where that information will be on each panel of the brochure. Then continue to answer more questions before you decide on a particular design.

\section{Consider and identify the audience}

Who is the audience for your brochure? Is it meant for English-speaking people or for immigrants or visitors from other countries? Is the brochure for experts or for people who are unfamiliar with the topic? Is the brochure meant for those who have the time to study it or for those who will glance quickly and move on? If your audience uses English as a second language, is less familiar with the topic, or is very busy, consider relying on visual images to carry your message. Steps highlighted with bullets or numbers may be more appropriate than complete sentences and paragraphs.

What else do you know about your audience? Many homeowners are older and more conservative than renters. Retirees may care about leaving the planet in good shape for future generations. What will motivate your audience to pick up the brochure and read it? Test your generalizations on a pilot group. Ask what they care about and which of several messages means the most to them.

Your text should be clear and understandable to your audience. If you are reaching the general public, write for an $8^{\text {th }}$ grade reading level. Use shorter sentences and common vocabulary. Use active verbs and action images. Do not use slang or the jargon of your field of expertise.

\section{Paper}

A brochure, pamphlet, or leaflet is a single, folded piece of paper. The typical format is a standard size of paper ( $8.5 \times 11$ inches) folded in thirds. A legal piece of paper ( $8.5 \times 14$ inches) is often folded in fourths, with either an accordion fold or a double-fold. They can be designed in a horizontal 
Landscape format, to be opened from left to right, or vertically, in a Portrait format and read down the page.

To decide on the size and the fold, consider:

- Will this brochure be distributed through the mail? Will it be mailed directly or inserted in an envelope? How big is the envelope? One option is to mail it directly, with one panel used for the address label.

- Will this brochure be picked up from a distribution rack? How big is the slot and how much of the front panel will be hidden?

- Is a response panel required? How much information will be included on it? Can it be reduced to fit into one panel or should an insert card be used?

\section{Budget}

Budget is an important determination when designing a brochure. Find out how much money is available for artwork, printing, and distribution and also if any parameters have been determined, such as quantity, size, or color. Once you have a basic idea, contact several local printers and ask for a price quote. Do this by visiting a few of the printers and seeing what printing papers they have available; review their costs. At this point you will not know how many pictures or colors you will have, but it is still helpful to have an initial conversation so that you do not overdesign for the available budget. As you develop your design, go back to the same printers and ask for an updated quote. It is always useful to develop good communication with your printer to avoid costly mistakes.

As a general rule, the use of more color and glossy (coated) paper means more expense, but these options do not guarantee an effective brochure. Success is the result of a good design, which may be just as effective with plain paper, one ink color, and a small budget. Many colors and types of recycled papers are readily available at a reasonable cost.

\section{Printing}

How many copies do you need? You can print 250 or 250,000 . Any quantity below 250 is best printed on a high quality commercial copier. At somewhere between 250 and 500, it becomes more cost efficient to have the brochure commercially printed. There is not much price difference between 500 and 1,000 brochures. In general once the press is set, you are only charged for paper. So, if the brochure is going to be something that will be used with no changes over a long period of time, it may make sense to print a larger quantity now and store them. The larger the quantity, the larger the printer's capacity should be. If you plan to print 10,000 brochures, go to a printer who has a large press to handle this quantity. A printer with a smaller press may charge more as the job will require more press time. Also, try to obtain quotes in writing. Be sure to give the same specifications to all the printers for easy comparison.

\section{Paper selection}

A printer can also help you decide what paper is economical for your brochure. Delivery schedule, due date, and price of the paper should be considered (Laundry and Vignelli 1991).

Paper is either coated or uncoated. Coated or glossy stock is better for printing photographs with fine details, but it is more expensive and does not hold up as well to the rigors of mailing. Uncoated stock is typically less expensive, more sturdy, and reproduces large graphics and text satisfactorily. Textured paper (a type of uncoated stock) can add to the visual appeal of the brochure but can also interfere with readability. To maintain readability with patterned paper, make sure the pattern is small and not the same color as the text.

Paper comes in two thicknesses: text (standard copy paper) and cover (standard card stock). It is manufactured in a variety of weights (20 pound, 50 pound, etc.). The weight refers to 1,000 sheets of 19 " $\mathrm{x} 25$ " text paper. The lighter weight, thinner paper is less expensive but more fragile. A heavier text weight or a lighter cover weight is usually the appropriate choice for a brochure. Discuss with your printer the alternatives available. He or she might have some 
"house stock" papers that are used for most printing jobs. It is often the most economical and also immediately available.

\section{Colored ink}

Will you use one ink, two colors of ink, or full-color in your brochure? Photographs often look better in full, four-color processing, but effective brochures can be made with one or two ink colors. Your choice of colors will depend on the capability of your printer - can he or she print color on site or must they ship the project to another printer? You will pay a handling fee if the job is sent to another printer. Small quantities are best printed one color.

"Full-color" means the printer uses four different ink colors (red, blue, yellow, and black) to achieve the full spectrum of color. Printers can use "spot colors" to print in a variety of colors of ink. Each color is the result of a formula and is referred to by a specific number; you can choose the exact perfect shade with a color chart from your printer. Several graphic programs also include color charts.

Unfortunately, MS Word ${ }^{\circledR}$ does not have the capability to separate each color in the four-process colors. If the final printing of the brochure is done commercially, then the printer will translate your file into another program that is set up to print to the four process colors. You can print directly from your Word file by 1) printing in one color or 2) separating the colors into different printed documents yourself. To accomplish the color separations "by hand," first print a color version for reference. Then select all the text of Color A and change it to white (use Font from Format menu and select White in the color section). Select all of Color B and change it to black. Print a copy that shows only Color B in black and save the file with the extension Color B. Now reverse the process, selecting all of Color $\mathrm{B}$ and changing it to white and printing a black version of Color A. The printed pages and the saved files will allow the printer to produce your design in 2 colors.

\section{Design}

\section{Composition}

Composition refers to the arrangements of elements and characteristics within a defined area. This arrangement should be visually pleasing, informative, and meaningful. A visual composition, like its counterparts in music, is effective when the elements are in harmony - when they make sense together.

\section{Relationship between elements and white space}

Space is perhaps the most important aspect of interaction in a composition. The areas between and around design elements are active parts in the overall composition, and they can be as dominant and important as the elements themselves (Bowers 1999).

Many people concentrate only on the element on the page, without realizing the importance of the white space. The most interesting layouts are achieved by creating an active relationship (Arntson 1993). This is done by altering the size and placement of the elements of the page, compared to the size and relationship of the white space. Figure 2 shows three examples of this relationship, from less active to more active:

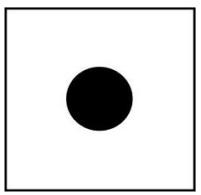

Figure 2.a

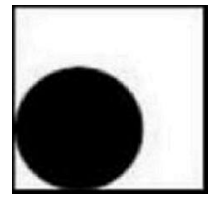

Figure 2.b

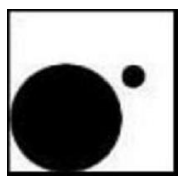

Figure 2.c

Figure 2. Examples of visual relationship, from less active to more active. 


\section{Balance}

The term balance refers to the distribution of the visual weight of design elements (graphics and text). The lack of balance can create confusion or irritation. Balance can be achieved through symmetry, when the design elements are mirrored on either side. A more dynamic form of balance is an asymmetrical design, when the design elements vary in density and distance but still carry the same visual weight; the right and left sides of the composition are not an exact mirror picture. White space is just as important as the design elements because it gives the eye a place to rest while viewing the whole page.

\section{Attention and hierarchy}

A design must capture and focus attention to communicate a message. The arrangement of design elements, the use of lines, and the choice of sizes will lead the eye in a particular direction. Put the most important paragraph or graphic at the focal point of the design.

You can capture attention by creating a hierarchy of design elements - making some stand out more than others - by contrasting the weight and shape of key elements. Headlines or important text can be set in a bolder, larger point size than the regular text. Lines can isolate or connect elements. Hierarchy can make a composition more engaging and help the viewer know which elements belong together (See Figure 3). Most successful designs rely on a carefully juggled balance of similarities and contrasts. There are two considerations in setting up balance through contrast: weight — the strength or dominance of the visual element, and direction - the way the eye is drawn over the layout (Arntson 1993).

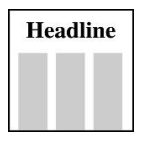

Figure 3.a

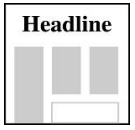

Figure 3.b

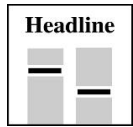

Figure 3.c

Figure 3. From simple to complex.

\section{Color}

If you are designing a brochure with color, consider the meaning that each color will create. Color can represent things as they are (such as green trees) or can connect elements together (such as all the subheadings). If you use visually pleasing colors, the design will be balanced and pleasing. Contrasting colors may create energy or irritation. Some colors carry cultural or symbolic meaning, such as red for anger, blood, or war in North America.

\section{Layout design}

Because a brochure is unfolded several times as it is read, you can tell a story, with each panel providing additional information. The front panel is usually a teaser that lures the reader inside. The next panel sustains interest and develops a theme, while the inside spread of panels holds the bulk of the information. The back panel is for a mailing label or explains to readers how to get more information.

The brochures in Figures 4 and 5 use color photographs and headlines on the cover to attract attention; they do not provide detailed information. One photograph is usually more powerful than several.

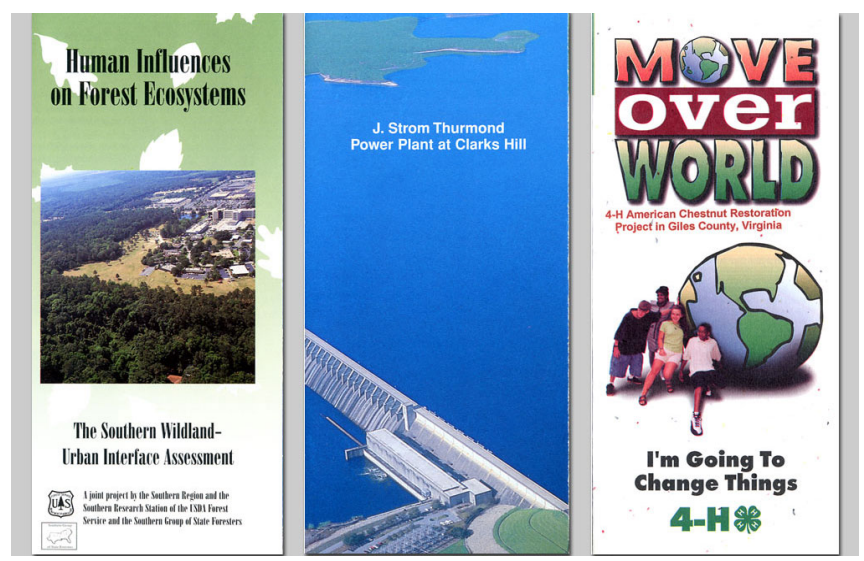

Figure 4. Images and text create different messages on brochures. Select the style that is most appropriate. 


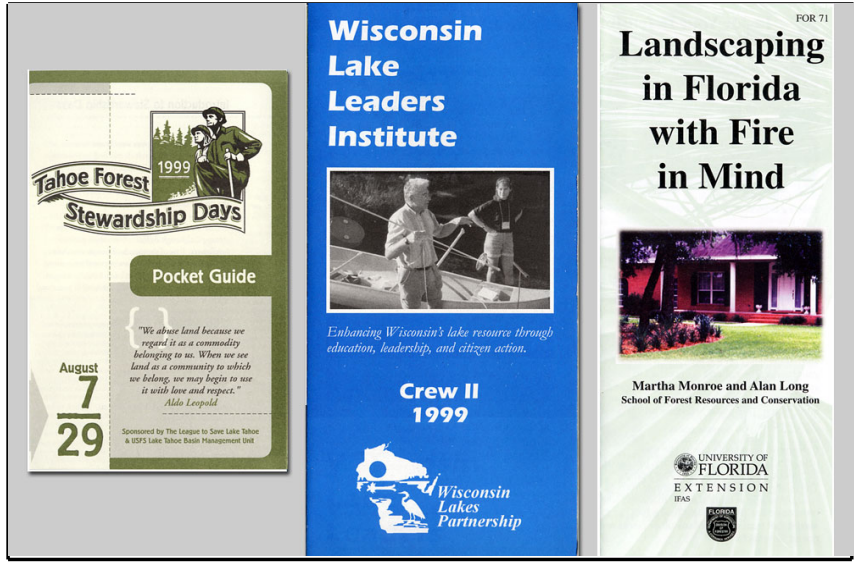

Figure 5. Photograhs and artwork attract attention on brochures.

\section{Orientation and margins}

The first step in designing a brochure is to choose the paper orientation and set the margins. In MS Word $2000^{\circledR}$ go to File and select Page Setup. Click on Paper Size and decide which orientation you wish to use: Landscape (horizontal) or Portrait (vertical). Then, set up the page margins, keeping at least one-half inch on all edges. This is the typical limit of printing presses and copiers. Do not switch orientations (from landscape to portrait) after the design is completed or within the same brochure. It is often helpful to make a mock-up of the actual brochure and work with it in your hands before doing a detailed design. See where the different types of information best fit on which panel. After you have done this and have a clear understanding, then you can start developing the layout. When selecting paper orientation specifications and margins, be sure to check Apply to Whole Document.

\section{Type of information - layout design}

Designers use an underlying grid system to provide a visual reference to a composition's organizational structure. The grid allows for the appropriate placement of components and ideas and helps create meaning and sense of continuity. A vertical grid is formed by the use of columns. Columns are typically used in a brochure to create parallel panels. If you have a lot of short phrases or bulleted points, not full paragraphs, use several narrow columns. If your text has large areas of continuous text, a wider design of two columns would be more appropriate. Consider using both, with a wide column above three small ones, if you need it. Columns are usually of equal size, but a good design can include narrow and wide columns. For symmetry, keep the same column layout for both sides of the brochure.

A grid also provides a horizontal structure in the brochure. Dividing your page into thirds or fourths gives you several levels on which to place pictures or typographic elements such as pull quotes. Pull quotes are short phrases that summarize adjacent text. For example, if you divide the horizontal page in fourths, you will have an imaginary line every 2.125 inches. So, at that measure (look at the side rule, Page Layout View), you can align the top of the pictures or the top of the pull quote boxes. It makes the layout more uniform and better organized.

\section{Adding text}

MS Word ${ }^{\circledR}$ allows you to import text from many programs, as well as to cut and paste directly into your document. When importing text, you can have it flow across the whole width of the document unformatted, or you can setup your columns and formats and then insert the text. Be sure to save often as you work on the file.

\section{Typography}

\section{Typography}

Styles (or style sheets) are mini-files containing your formatting options. Styles save time by allowing you to assign multiple formatting selections with a single keystroke. Styles also ensure page-to-page consistency. See the section below for more information on Styles.

Most brochures rely on text to convey the message. Choose a style font to create the appropriate look and feel for your brochure. Do not use a True Type font - those with a double T next to the name. They do not translate well from one machine to another if you are going to a commercial printer for final output of your file. However, they can work if you use the same machine and laser printer for your final output. 


\section{Readability and legibility}

The term readability refers to how easily a large amount of text can be read. The shape of the letters can help improve readability. Serif fonts are thick and thin letters that have a stroke projecting off the letter at its bottom or top, and they are commonly used for large amounts of text. Newspapers are set in a serif font, such as Times Roman and Palatino.

\section{Legibility refers to how instantly recognizable} individual letters are, a quality that makes short bursts of type (such as headlines) easier to read. Sans serif fonts are often used in places where you want to pull readers into the main text, such as headlines or pull quotes; they are letter-forms without a serif. Helvetica and Futura are sans serif fonts (Williams 1994).

\section{Font size}

Type size is measured in points up to about two inches high. When selecting a type size, consider your audience. Brochure text should be at least 12 points for reading ease. Larger type sizes are easier to read, but only to a point; they may also increase the number of hyphens, which interferes with legibility. As a rule, keep hyphenation to less than two per paragraph.

\section{Line-spacing and word-spacing}

You can control the space between paragraphs, the space between lines, and the space between characters by altering the parameters of your style sheet. You can vary the spacing within a brochure to set off important paragraphs or call attention to a pull-quote, without inserting additional paragraph breaks. Unless you have a design reason, keep these variables set to "normal" and keep them consistent throughout the brochure.

\section{Alignment}

There are four basic alignment categories: left, center, right, and justified. The left and right alignments create one straight edge and one ragged edge. Right alignment is usually difficult to read. Centered text is often found in headline copy but rarely used for formatting the whole text of the brochure. The justified text creates two straight edges, which usually means odd spacing between words if the column width is small. This format is commonly used in newspaper layout.

\section{Hierarchy on the page}

Headlines and subtitles are more visible and larger than the rest of the text. This creates a visual order so the reader finds the information in a logical sequence. The same should happen with the text. If some of the text requires immediate attention, or should be read first, you can establish a design hierarchy to make sure the reader follows it. One way to establish this visual order is to: 1) change the size of the type; 2) change the space above and below the paragraphs; 3) use lines, dots, or other design elements to divide the page.

\section{More on design elements}

- Initial caps or drop caps are one of the elements that may be used in a brochure, especially when there is a lot of text and few pictures. They should be much larger than the following text and set in a font that matches the headline but contrasts with the text. Drop caps should closely relate to the text they introduce. Be sure to align the baseline of the drop cap with one of the lines of the paragraph and to wrap the surrounding text tightly to the initial cap. Do not use a one- or two-letter word in the beginning of the first sentence, if you use the drop cap; rewrite the sentence if necessary (Parker 1994b). Try setting the initial caps and headline in Helvetica Bold and the text in Times Roman. See Figure 6. Select Drop Cap from the Format menu, and follow the directions of the dialog box.

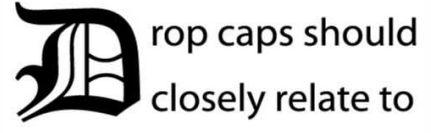
the text they introduce.

Be sure to align the baseline of the drop cap

Figure 6. Drop caps add variety to the layout. 
- Pull quotes offer another opportunity to draw the reader's attention and add interest to the page. Set them in a different font and at a significantly larger size than the body text. See Figure 7. Select Text Box from the Insert menu and type or insert the text for the quote. Use Paragraph and Character dialog boxes to set up the format.

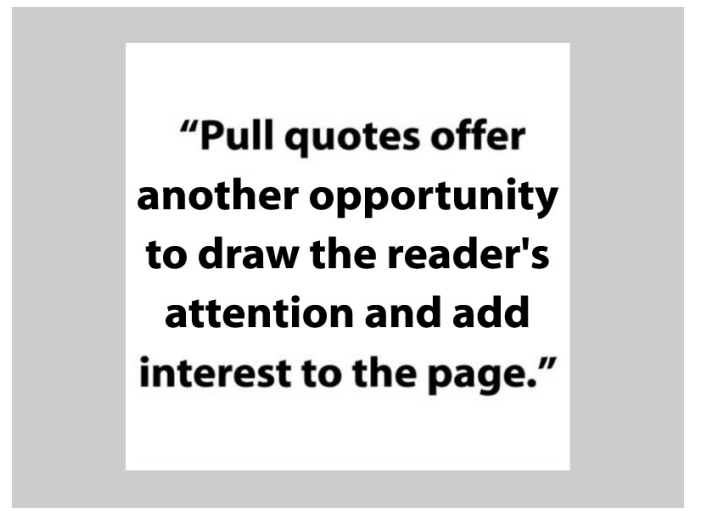

Figure 7. Pull quotes draw attention to a specific sentence or paragraph.

- Dingbats are ornamental design elements. They are set as type and should be sized at a minimum. They are often used in place of bullets, if they do not overpower the message of the text. See Figure 8. Consider screening the dingbat, by selecting a color fill and choosing a lighter shade, to make it a bit more elegant and less offensive. Bullets also help with readability. MS Word automatically creates bullets and numbers for a selected text. To do this, highlight the text and select Bullets and Numbering from the Format menu. To customize the bullets with dingbats or other symbols, highlight one of the options for bullets. Click on Customize, Customized Bulleted List. Then click on Bullet. The Symbol dialog box appears; click on the down arrow in the Font box to locate Wingdings, and choose the desired bullet. To change the size, use the Font button on the Customized Bulleted List.

- Rule lines also help organize the visual space on the layout, bring attention, or group elements. See Figure 9. Use each design element with caution; each element should have a precise function and add to the overall message. Rule lines can be added by selecting Borders and Shading from the Format menu. You can also set rule lines by clicking on the Top, Bottom, Left, and Right Border buttons on the Alignment option of the Standard toolbar. You can
Dingbats are also ornamental design elements.

They are set as type and should be sized at a minimum.

Figure 8. Dingbats help lists stand out.

also use the Drawing toolbar. The line size and style can be readily modified with the Drawing toolbar.

Rule lines help
organize the visual
space on the layout,
bring attention, or
group elements.

Figure 9. Rule lines help organize text on a page.

- Reverse text (white text on black background) makes a very strong visual statement, but it is hard to read. See Figure 10. Reverses work best when limited to a few large words, such as headlines or subtitles (Parker 1994a). To create reverse text select Text Box from the Insert menu, or from the Drawing toolbar click on the Text Box button, and type in the text or insert it from a file. Change the Fill for the box from Clear to Black. You can do that by double clicking on the box, or by clicking on the Fill Color button on the Drawing toolbar. Change the text to white, under the text icon, creating reverse copy. Use the Font, Paragraph, and Text box dialog boxes to format it.

\section{Images}

Whether you are working with photographs, original illustrations, or clip art, make sure they are appropriate to your document in both subject matter and style. Use whatever methods are available to enhance the pictures, such as cropping a photograph or brightening the contrast. 


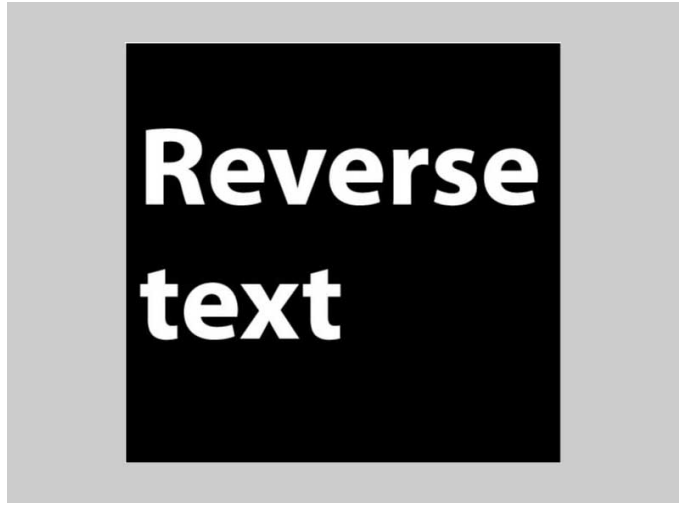

Figure 10. Reverse text can be very dramatic and effective on the page.

Pay attention to both the size and placement of pictures. If they are attractive and meaningful to your brochure, do not be afraid to make them big. Purely decorative items should be kept small. Take advantage of your grid to place pictures so that they fit cleanly into the layout. If the pictures break the layout, do so intentionally, at least three times, within the layout. This allows the reader to understand the underlying visual rhythm. For example, if you only break the grid in two areas, where a picture is outside the layout or grid margin, it may look like a mistake or a misalignment. If it is done three times, the reader will understand it as part of the design (Parker 1994a).

\section{How to scan pictures for final output}

- Use a scanning device and scanning software to digitize a slide, flat artwork, or a photograph so that it can be read, displayed, edited, and printed by a computer.

- Scan mode or image type: select black and white line art (no grays), black and white photograph (grays), or color. A picture scanned in color will be approximately three times larger in file size than the same picture in black and white.

- Choose a format for saving the scanned image depending on whether you produce the final camera ready copy (a final high resolution laser print), or you give the file to a printer. Common file formats for pictures are: Windows Bitmap (.bmp), TIFF Bitmap (.tif), and Encapsulated PostScript (.eps). Lower resolution formats are: JPEG File Interchange format (.jpg) or Graphics Interchange Format (.gif). Your final image for the printer should be a TIFF file at $300 \mathrm{dpi}$.
- Choose the minimum resolution necessary to obtain the best print for your brochure. Scan resolution is measured in pixel per inch (ppi). The higher the resolution, the better the scan, and the larger the file size. A picture with a higher resolution will require more disk space and will take a longer time to print. You can create two versions of your picture, one to work with in the layout and one for the printer to use. However, most commercial printers will prefer doing the high resolution scans on their own equipment, as it is far better than most personal-use scanners. If this is the case, at the time of a proof, make sure your printer replaces the picture with a higher resolution version of the original.

\section{How to combine images with typography effectively}

Remember that the text and images should each bring more clarity and strength to the overall message in the brochure. To determine if you are successful, remember your initial purpose and audience assessment. Pilot test the brochure with the audience, friends, or associates.

\section{Setting up the brochure}

\section{Before you begin}

Start by labeling a series of High Density floppy disks, a RW-CD, or a Zip disk with your name. Insert one disk and create a folder for your images and pictures. This system keeps everything in one spot and makes it easy to identify and retrieve the right material.

As a precaution against computer error and disk malfunction, make a copy of your disk.

\section{Start from scratch}

From the File menu, select New. Select the General tab, and then double-click the Blank Document icon or click the New Document button from the Standard toolbar.

Set up page orientation and margins by selecting Page Setup from the File menu. The dialog box will have different layers to set up both margins and orientation. Again, keep in mind that you do not want 
to select different orientations or margins for each side of the brochure.

As soon as possible save your file to your disk, and remember to save your work regularly.

\section{Create columns}

Columns are the basic alignment for the text and the images. From the Format menu, select Columns, or from the Standard toolbar menu click the

Columns button. If you are using the Columns dialog box, it may be easier to set up the columns in a more specific and precise way. If you have already imported text, click Select All from the Edit menu, and then select the desired options from the Columns dialog box.

\section{Import text}

Select File from the Insert menu, and select your text file to be imported. The text will start where the cursor is on the page, usually at the top left, and will automatically flow into the designated columns. In Word you can also type text, and you will see it flow into the designated columns as you type. This may be a good time to delete any page breaks you may have had in the original document, because they will disrupt the flow of text into the columns. You can also open the file and Cut and Paste text into your brochure.

\section{Change the color in text and numbers}

There are times you may want to change the color of text, numbers, and dingbats. To do so, select the text, click the arrow next to the Font Color button, and select the color you want. To apply the color most recently used for text, click Font Color on the Formatting toolbar.

\section{Spell check}

This is a great time to run the spell checker for the whole document. From the Tools menu select Spelling and Grammar, or click on the Spelling button on the Standard toolbar.

\section{Using styles}

A style is a set of formatting characteristics that you can apply to text in your document to change the appearance quickly and consistently. When you apply a style, you apply a whole group of formats in one simple task. For example, you may want to format the headline for your brochure. Instead of taking three separate steps to format the headline as 24 point, Times Roman, centered-alignment, you can achieve the same result in one step by applying the headline style to each of your existing headlines.

A paragraph style controls all aspects of a paragraph's appearance, such as text alignment, tab stops, line spacing, and borders, and can include character formatting. Again, the best way is to modify the paragraph style. You can do this by selecting Style from the Format menu.

A character style affects selected text within a paragraph, such as the font and size of text and bold and italic formats. Characters within a paragraph can have their own style even if the paragraph style is applied to the paragraph as a whole. This may be necessary to bring special attention to part of the text within the paragraph.

By going to the beginning of the document or the text, you can set your styles for the whole brochure. Let's assume that you have a headline, a number of subtitles, a couple of pull quotes, and the actual body of text.

Select Style from the Format menu. In the dialog box you have a choice of creating new styles or applying the program default styles. Select Modify and you can set the font style, the point size, the alignment and other properties. In addition, you can set up a shortcut key for each style. The style name is displayed within the Standard toolbar. These modified styles will be the styles for your specific file for this brochure. If you have several subtitles that may have different levels, you can name them for example: subtitle 1, subtitle 2, etc., and each can be based on one single font style, but at different sizes, or one is regular type (Roman), and the second one is italic. 
When setting up the styles for the body text, you may want a different one for each first paragraph that comes right after a headline or a subtitle. That very first paragraph should not be indented. In this case, you can name it first body, and the style for rest of the body text can simply be body text.

Once you have set all the styles, click OK or Apply. You can always go back to Modify and change the styles as you see fit in the course of the brochure development.

Now that you are ready to apply the styles, select the text and select the style from the pull-down menu on the Standard toolbar.

\section{Importing pictures and clip art}

The best quality digital images are obtained from scanning the original film (slides or negatives) or output from high resolution (>3 megabyte) digital cameras. If you routinely need to scan images, $35 \mathrm{~mm}$ film scanners that have resolutions of 3000--4000 dpi (dots per inch) and a dynamic range of 3.6--4.0 will do a good job. Commercial scanning of images for typical brochure size files costs about $\$ 5$ per scan. Larger, more detailed scans can be obtained with drum scanners, but a drum scan costs $\$ 25-\mathbf{- \$ 1 0 0}$ and the higher quality won't be noticeable on small brochure images. Flatbed scanners perform well with line art and can produce satisfactory results with photos if the surface isn't textured and they are at least as big as they will be in the brochure.

Images should be scanned at a resolution that will produce a 300 dpi print file and saved in an uncompressed format such as TIF. Most graphic programs work in computer screen resolution units called pixels. For practical purposes a pixel is equivalent to a dpi. If the brochure image is to be 3 inches $\mathrm{x} 4$ inches, then the scan needs to be 300 dpi $x$ 3 inches $=900$ pixels wide by 4 inches $\times 300$ dpi $=$ 1,200 pixels high. Film scanners set at the highest resolution will generally produce files much larger than you need but they can be easily resized. File sizes can be reduced without a noticeable loss in image quality, but generally they can't be enlarged. If the line art or photo prints are the same size as in the brochure, then flatbed scanning resolution is simply set to $300 \mathrm{dpi}$. If the originals are larger, you should still scan at 300 dpi and reduce the file size in a graphics program. There are many good graphic programs available to work with images, but Adobe Photoshop ${ }^{\circledR}$ is a graphic industry standard. Although the program is expensive, time spent learning Photoshop will be well invested. The artistic possibilities with Photoshop tools are limitless and dramatic improvements to your scans can be achieved.

MS Word ${ }^{\circledR}$ inserts imported pictures from the program's Clip Art file as a floating picture. This allows you to make changes to that image. To import a picture from the Clip Art file, select Picture from the Insert menu, and select From Clip Art.

To import any picture or graphic-file type, choose Picture from the Insert menu, select From File, locate and select the picture file, make sure to check the box for Float over text (located at the bottom right area of the dialog box), and click the Insert button.

To make any changes to the picture, select it and choose Picture from the Format menu. A Format Picture dialog box will appear, with several tabs allowing you to do the following: add/change Colors and Lines, change Size, change Position, change Wrapping (flowing text around the picture) and change Crop (keeping the same size but cutting out part of the picture). Experiment with the Text Flow options by clicking on the picture. In addition, you can nudge an image in small increments, by selecting it and pressing the Control key and at the same time the arrow keys: left arrow key, the right arrow key, the up arrow key, and the down arrow key; this moves an object in 1-pixel increments.

The inserted picture is now part of the document, and as such it is subject to the paragraph's characteristics. If only part of the picture is showing, select the graphic, click Paragraph on the Format menu, and then click the Indents and Spacing tab. In the Line Spacing box, click Single. If you select Exactly in the Line Spacing box, be sure to increase measurement in the At box to the height of the graphic. 
If you see the text but not the picture that you have imported, make sure that you have turned on the display of graphics. On the Tools menu, choose Options, and then click the view tab. Clear the Picture Placeholders check box.

\section{Scaling pictures}

Placed pictures will have anchor points at the four corners. Select the picture and click on one of the corner anchor points with the Shift key to manually proportionally scale the pictures. You will see the cursor change into a double arrow, which means that you are making a size change. At the same time press the Shift key to make the change in proportional scale. Or use the Picture dialog box from the Format menu to scale mathematically and proportionally.

\section{Using a grid for layout alignment}

Earlier we discussed how a grid may help with alignment for pictures or drawn objects. The drawing grid is an invisible network of lines that helps you align objects. As you drag an object, Word pulls it into alignment with the nearest intersection of gridlines. By default, gridlines are not visible on the screen, but you can display gridlines to view the drawing grid. To view gridlines, display the Drawing toolbar by clicking Drawing. On the Drawing toolbar, click Draw, and then click Grid. Select the Display Gridlines on Screen check box. To change the spacing between the drawing gridlines, click Draw from the Drawing toolbar, and then click Grid. In the Horizontal and Vertical Spacing boxes, enter the spacing you want. You can also use the same dialog box to change the starting point for gridlines, relative to the edges of the paper. To turn off the drawing grid, clear the Snap objects to grid check box.

\section{Special applications}

In many cases, registration forms and agendas will occupy one or more panels of the brochure, becoming a separate design element. Determine what information is required and how much space it would need. Rule lines help to set these items apart from the text.
Within the form, make sure the flow of the text is logical and keep demographic questions together, separated from food preferences or arrival time. Ask a co-worker to review the form to make sure it is understandable. The text point size could be smaller than the remainder of the brochure text, but no smaller than 8 point. Select the same font you used for the brochure text. Lines should be thin and spaced well enough for respondents to write the information. Boxes help keep the form clean and direct users to fill-in or check-off options. Shaded backgrounds of $20 \%$ will help call attention to a section (Schwebach 1994).

If you wish to have the form perforated (so the reader can easily tear the form away), it will add an additional cost to the printing charge. If you are working with a tight budget, you may want to indicate by a thin dotted line that the reader can tear or cut the form off the main body of the brochure.

\section{Taking your brochure to the printer}

When you have completed your brochure, take to the printer a paper mock-up version, the disk, a copy of the printing estimate, and the original artwork so it can be rescanned, if necessary. Make sure the printer has matching fonts or that your disk contains the final complete brochure with a folder with all the scanned pictures that you have used and the fonts.

Take the time to walk through the whole brochure with the printer to make sure all of your expectations are clear. Confirm your quote, quantity, paper, colors, payment, delivery date, and place of delivery. Make sure that the printer has all the information necessary to get in touch with you. In the end, communication with the printer or any other vendor makes the design a total success.

\section{Problems to avoid}

\section{Misspellings}

Misspellings are unacceptable. Use the spell checker on all of the text, including the text that is imported from other publications or files. In addition, if using original copy, click on Grammar from the Tool menu. Because spell checker can not identify correctly spelled but incorrectly used words, you still 
need to read the document carefully for common mistakes (e.g., from and form).

\section{Creating confusing layouts}

If your layout is not clear, the reader will not know where to start reading, what the order is, and which is the most important information. Give your brochure to friends or associates to proofread. If there are problems, you will need to go back to the initial layout stages and start over.

\section{Using too many fonts}

As a rule, in a single publication, two different fonts is the maximum you should use. You can just stay with a single family, for example Times Roman, and have headlines and pull quotes in bold and the text copy in regular, with captions in italic. This is all a single font. If you wish to use a second font, mix a serif with a sans serif font, but to avoid confusion do not use every style option (bold, italic, and regular).

\section{Using different text alignments}

Using too many alignment styles will also create confusion in your brochure. If you center all the headlines and subtitles, you can still have the text copy aligned to the left or justified. Do not use two different alignments for the text copy.

\section{Incorrect punctuation and spacing}

For proper typesetting, do not use hard return at the end of each line or between paragraphs. Use the spacing option for after paragraphs in the Format menu.

MS Word ${ }^{\circledR}$, as a default, uses "smart quotes," open or closed quotes, rather than inch marks (except in measurements) and apostrophes rather than foot marks. If, for any reason, your document is not formatted with smart quotes, you can do the following to change it. From the Tools menu choose AutoCorrect. Select the Change from Straight Quotes to Smart Quotes and choose OK. You can also use the Find/Replace dialog box to change the ones you have not already done. In addition, they are available under the Special Character option.
Replace the commonly used two hyphens (--) with an em dash (-) (ALT 0151) to introduce and close parenthetical phrases, in other words, use the em dash in place of parenthesis. Use an en dash (-) (ALT 0150) when indicating a range or a duration (January-February, 5:00-7:00 pm or 2000-3). Use hard (nonbreaking) hyphens to keep names and dates from splitting across two lines (Parker 1994b).

\section{Unnecessary spacing}

Regardless of how you learned to space after a period, use only one now. Do not indent the first line of a paragraph following a headline or a subtitle; indents are only necessary within columns of text. Hyphenate left-aligned text to avoid differences in line length and distracting shapes (Parker 1994b). With justified text, adjust the word spacing to reduce distracting gaps in the text.

\section{Widows and orphans}

Avoid widows and orphans because they refer to isolated line endings and dangling words, either at the end of a paragraph or column. Widows is a single short line, when paragraphs end on the first line of a page or column. Orphans are created when the first line of a paragraph begins at the last line of a page or column. Avoid them by checking the square on the Paragraph Dialog box on the Format menu.

\section{Creating space in a line}

Some people are afraid to use tabs instead of the space bar to create appropriate alignments or indentations. Tabs create a much more professional result, and they are more time efficient. You can easily set tabs by selecting Tabs from the Format menu.

\section{Gray pages}

Gray pages are the result of too much type, not enough white space, and/or lack of typographic contrast between various elements. Readers should be able to identify quickly headlines, subheads, body copy and captions from your hierarchy. Avoid font, type size, and type style choices that are only slightly different from each other; this creates confusion or boredom (Parker 1994b). 


\section{Insufficient margins}

White space at top, bottom, and sides of a page makes it look more inviting and easier to read.

\section{Inappropriate column width}

Long sentences of small type in columns are tiring to read because each requires several left-to-right eye movements. In addition, readers may get lost at the end of the line and return to the beginning of the wrong line. Narrow columns of justified text do not offer enough opportunities for words to be properly spaced. As a result, lines containing a few long words could have large gaps between words or excessive hyphenation (Parker 1994b).

\section{Inappropriate borders}

Page borders should reinforce the text, not distract from it. Borders can create barriers that discourage movement to the next panel (Parker 1994b).

\section{Overuse of rule lines or design elements}

Rule lines should emphasize the end of one topic and another the beginning of another topic, as above a headline. Below a headline, the same line can present a visual barrier (Parker 1994b).

\section{Infringing copyrights}

Copyright laws cover original visual images and text. The only way not to infringe on them is to use original text and art (photographs and illustrations), to get permission from the original artist to use his/her work, or to use clip art (noncopyrighted material). Computers have blurred the lines of what is "original art", however, using somebody else's image and making slight changes to it, is still infringing on copyright laws.

\section{Summary}

A successful brochure is a purposeful blend of good design and meaningful text. Design encompasses many different elements which can be altered in numerous ways. Many different designs can lead to a successful brochure if one is creative enough to put new layouts on paper. This publication provides only the basic concepts of design for the beginning brochure designer, but opens a world of possibilities.

\section{Literature Cited}

Arntson, Amy E. 1993. Graphic Design Basics. Fort Worth, TX: Harcourt Brace Jovanovich College Publishers, USA.

Bowers, John. 1999. Introduction to Two Dimensional Design: Understanding Form and Function. Canada: John Wiley \& Sons.

Laundry P., and Vignelli M. 1991. Graphic Design for Non-Profit Organizations. New York, NY, USA: The American Institute of Graphic Arts.

Parker, Roger C. 1994a. 10 common mistakes to avoid. Technique, Nov/Dec: 64.

Parker, Roger C. 1994b. Page layout basics. Technique, Nov/Dec: 57-62.

Schwebach, Lynn S. 1994. Design electronic forms. Technique, Nov/Dec: 32-33.

Williams, Robin. 1994. Choosing Typefaces. Technique, Nov/Dec: 17-28. 